\title{
Review
}

\section{Mature neurons: equipped for survival}

\author{
AJ Kole ${ }^{1,2}$, RP Annis ${ }^{2,3}$ and M Deshmukh*,1,2
}

Neurons completely transform how they regulate cell death over the course of their lifetimes. Developing neurons freely activate cell death pathways to fine-tune the number of neurons that are needed during the precise formation of neural networks. However, the regulatory balance between life and death shifts as neurons mature beyond early development. Mature neurons promote survival at all costs by employing multiple, often redundant, strategies to prevent cell death by apoptosis. This dramatic shift from permitting cell death to ensuring cellular survival is critical, as these post-mitotic cells must provide neuronal circuitry for an organism's entire lifetime. Importantly, as many neurodegenerative diseases afflict adult neuronal populations, the survival mechanisms in mature neurons are likely to be either reversed or circumvented during neurodegeneration. Examining the adaptations for inhibiting apoptosis during neuronal maturation is key to comprehending not just how neurons survive long term, but may also provide insight for understanding how neuronal toxicity in various neurodegenerative diseases may ultimately lead to cell death.

Cell Death and Disease (2013) 4, e689; doi:10.1038/cddis.2013.220; published online 27 June 2013

Subject Category: Neuroscience

Facts

- Programmed cell death, or apoptosis, is active and necessary during early nervous system development.

- Mature neurons become strikingly resistant to cytotoxic stimuli following the developmental stage.

- Brakes are engaged both pre- and post-mitochondria in the apoptotic pathway in mature neurons.

- The use of multiple, redundant mechanisms to inhibit apoptosis probably provides mature neurons with longterm survival capabilities.

\section{Open Questions}

- Are there other undiscovered mechanisms engaged in mature neurons to inhibit cell death?

- What upstream molecular cues instruct neurons to become mature and engage cell survival mechanisms?

- Do mature neurons revert to a phenotype similar to immature neurons during neurodegeneration?

The precise regulation of basic biological processes, such as cell growth, proliferation, differentiation, metabolism, and cell death, is critical for cellular function. Equally important, cells must also have the ability to adapt these biological pathways in response to developmental or environmental cues. For example, in neurons, although the cell death pathway is remarkably active during early development, it becomes highly restricted as neurons mature. This striking adjustment to the regulation of cell death during maturation is a critical event for neurons, as these post-mitotic cells must survive and perform vital functions for an organism's entire life.

Programmed cell death, or apoptosis, is normal, necessary, and occurs in surprisingly large numbers during nervous system development. ${ }^{1}$ Such massive developmental death is critical for the remarkably complex task of establishing a perfectly wired nervous system. This task is accomplished by generating an abundance of neurons and eliminating those that are developmentally transient, fail to properly migrate, or are not matched to survival factors provided by afferent inputs. ${ }^{2}$ For example, in the peripheral nervous system (PNS), nearly twice as many neurons are initially produced as are eventually found in the fully developed nervous system. ${ }^{1}$ In order to survive, developing neurons must reach and innervate their appropriate target cells, which supply critical survival promoting trophic factors, but in limiting amounts. Those neurons without trophic factor support undergo apoptosis, thus elegantly generating a functional, matched network of neurons, and their target cells. ${ }^{3,4}$

Importantly, once the nervous system is fully developed, neurons can no longer afford to remain susceptible to apoptosis, as these post-mitotic cells have limited regenerative potential and must live long term. Therefore, it is imperative for neurons to carefully inhibit the apoptotic

\footnotetext{
${ }^{1}$ Department of Cell Biology and Physiology, University of North Carolina, Chapel Hill, North Carolina, USA; ${ }^{2}$ Neuroscience Center, University of North Carolina, Chapel Hill, North Carolina, USA and ${ }^{3}$ Curriculum in Neurobiology, University of North Carolina, Chapel Hill, North Carolina, USA

${ }^{*}$ Corresponding author: M Deshmukh, Department of Cell Biology and Physiology, University of North Carolina, 7109E Neuroscience Research Building, Box 7250, 115 Mason Farm Road, Chapel Hill, NC 27599, USA. Tel: 919843 6004; Fax: 919966 1050; E-mail: mohanish@med.unc.edu Keywords: programmed cell death; apoptosis; mature neurons; neurodegeneration; miR-29; Apaf-1

Abbreviations: ALS, amyotrophic lateral sclerosis; Apaf-1, apoptotic protease activating factor 1; AVCN, anteroventral cochlear nucleus; BACE1, $\beta$-site APP-cleaving enzyme 1; BDNF, brain-derived neurotrophic factor; BH, Bcl-2 homology; CNS, central nervous system; DRG, Dorsal root ganglion; GDNF, glial-derived neurotrophic factor; HD, Huntington's disease; JNK, c-Jun N-terminal kinase; miRNA, microRNA; miR-29, microRNA-29; MLK, mixed lineage kinase; mSOD1, mutant superoxide dismutase-1; NGF, nerve growth factor; NMDA, N-methyl D-aspartate; PNS, peripheral nervous system; TUNEL, terminal deoxyribonucleotidyl transferase-mediated dUTP nick end labeling; XAF, XIAP-associated factor; XIAP, X-linked inhibitor of apoptosis protein

Received 06.5.13; revised 20.5.13; accepted 21.5.13; Edited by G Raschellà
} 
pathway as they mature. To implement this rapid switch from apoptotic susceptibility to strict inhibition, mature neurons appear to engage multiple mechanisms to inhibit the pathway of apoptosis. Failure to maintain these brakes on apoptosis is likely to increase the vulnerability of mature neurons to degeneration.

Indeed, evidence has been accumulating in the literature for over three decades supporting the concept that mature neurons of the PNS and central nervous systems (CNS) are markedly more resistant to cell death than developing immature neurons. In this review, we will begin with diverse examples that highlight the various insults against which neurons gain resistance during their maturation. Next, we will describe our understanding of the specific mechanisms utilized by mature neurons to strictly inhibit the intrinsic pathway of apoptosis. Finally, we propose that an important transition that occurs during neurodegeneration is the return of neurons to an immature state, thereby increasing their susceptibility to apoptosis. The intent of this review is to focus primarily on the dynamic changes in the apoptotic pathway that occur with neuronal maturation. The overall mechanism of apoptosis in neurons has been comprehensively reviewed elsewhere. ${ }^{2,5-7}$ Although the mechanism by which the apoptotic pathway becomes inhibited with neuronal maturation remains incompletely understood, this field is poised to take advantage of the many recent advances in our knowledge about apoptosis.

It should be noted that the timing of development varies between the different neuronal types and thus the point at which neurons become mature and resistant to apoptosis will be different for various neuronal types. ${ }^{1,2}$ However, despite these differences in the precise timing of neuronal maturation, a consistent theme that we highlight is that neuronal maturation is accompanied with increased resistance to apoptosis.

\section{Resistance of Mature Neurons to Diverse Apoptotic Stimuli}

The surprising observation that healthy neurons routinely die during normal development was first made by the laboratory of Erich Kallius, ${ }^{8,9}$ then more extensively explored by Viktor Hamburger and Rita Levi-Montalcini. ${ }^{10}$ Among the first evidence that young neurons are particularly susceptible to naturally occurring, trophic factor deprivation-induced cell death came in 1960, when Rita Levi-Montalcini and colleagues injected mice with an antiserum to the then recently discovered nerve growth factor (NGF). ${ }^{11}$ When NGF antiserum was injected into newborn mice, histological analysis of superior cervical ganglia showed loss of $97-99 \%$ of neurons compared with control. This technique, known as immunosympathectomy, resulted in the nearly complete destruction of the mouse sympathetic nervous system. These data highlighted the importance of NGF for sympathetic neuron development and survival. Perhaps just as striking, but overshadowed at the time, was the observation that in the only adult mouse injected with the same NGF antiserum, 34\% of sympathetic neurons remained viable versus control. ${ }^{11}$ The difference in response between young and adult mice was intriguing, but this observation was not followed up for more than 10 years. Subsequent studies confirmed that although
NGF antiserum in adult mice caused a pronounced reduction in cell size, as seen by electron microscopy, the survival of neurons was indeed largely maintained. This was most evident with the observation that although the functional capacity of adult neurons was initially reduced by injection of NGF antiserum in adults, as measured by decreased neuronal catecholamine synthesis and target organ catecholamine uptake, the neurons recovered once the anti-NGF injections were stopped. ${ }^{12-15}$

This phenomenon has also been recapitulated in vitro using a model of sympathetic neuron death induced by NGF deprivation. Nearly, $100 \%$ of young sympathetic neurons that are equivalent of post-natal day 5 (P5; neurons isolated from PO mice and maintained in culture for 5 days) undergo cell death by apoptosis following removal of NGF. In contrast, when these neurons are maintained in culture until P28 equivalent, they become strikingly resistant to apoptosis as less than $10 \%$ are now susceptible to the same insult of NGF deprivation. ${ }^{16-20}$ Likewise, sensory neurons from the dorsal root ganglia (DRG) have also been shown to become insensitive to NGF deprivation upon maturation. ${ }^{21-23}$ Interestingly, acquisition of this NGF-independent phenotype in DRG neurons is dependent on calcium signaling, as neurons matured in low calcium-containing media remain sensitive to NGF deprivation. ${ }^{24}$

Other developing neuronal populations that undergo cell death in response to deprivation of sensory input to afferent neurons also show age-dependent sensitivities. For example, in the developing auditory circuitry, neurons in the anteroventral cochlear nucleus (AVCN) undergo cell death if the cochlea is removed during a critical early post-natal period. ${ }^{25}$ This death is apoptotic as it can be inhibited with the antiapoptotic protein $\mathrm{Bcl}-2 .^{26}$ Studies have shown that cochlear removal in P5 mice causes approximately 60-80\% AVCN neuron loss, measured by histologic cell counting and terminal deoxyribonucleotidyl transferase-mediated dUTP nick end labeling (TUNEL) stain. Importantly, however, if cochlear removal occurs in mice that are just 1-week older, neuronal death in the AVCN is minimal. ${ }^{27,28}$

Intriguingly, the remarkable survival of mature neurons is seen not only when challenged with developmental cues, but also following axotomy, traumatic brain injury, hypoxia, or viral infection. For example, it appears that one of the most important factors determining whether a neuron will survive after axotomy is the age of the neuron. ${ }^{29}$ Although only $40 \%$ of motor neurons survive transection of the hypoglossal nerve in 1 -week-old mice, nearly twice as many neurons survive the same transection if performed on 3 -week-old mice. ${ }^{30}$ Interestingly, not only do a smaller number of mature neurons die after nerve transection, but the rate of neuronal death is also much slower after nerve transections in older animals. ${ }^{30-32}$ The correlation between neuronal maturity and survival after axotomy or crush injury has been shown in a variety of neuronal subtypes, including neurons of the rat facial nerve ${ }^{32-34}$ and dorsal root ganglia, ${ }^{35}$ retinal ganglion cells ${ }^{36,37}$ or following head trauma. ${ }^{38}$

In neonatal rat models of hypoxia and ischemia in vivo, a significant amount of neuronal death occurs by apoptosis, as evidenced by increased TUNEL labeling, DNA laddering, and features of apoptosis on electron microscopy. ${ }^{39}$ In addition, 
caspase inhibitors can prevent neuronal loss in this model, consistent with the observation that caspase-3, a protease and critical mediator of apoptosis, becomes active after neonatal hypoxia-ischemia. ${ }^{39,40}$ In contrast to the neonatal models, hypoxia-ischemia in progressively older rats results in markedly reduced caspase-3 activation with fewer cells showing morphological characteristics of apoptosis, indicating that apoptosis becomes more restricted with developmental age. ${ }^{40-42}$ Interestingly, although the apoptotic pathway becomes blocked with increasing neuronal age, neuronal viability is still affected upon severe hypoxiaischemia, with many neurons exhibiting features of necrosis rather than classical apoptosis. ${ }^{41}$

In response to viral infections, young and mature neurons also show differential capacity to survive. For example, intracerebral inoculation of Sindbis alphavirus causes a fatal encephalitis in neonatal mice and is associated with CNS toxicity. ${ }^{43}$ Interestingly, although Sindbis virus causes $100 \%$ mortality within 8 days when inoculated in the cerebrum of P1 mice, the same viral challenge in 4-week-old mice causes an asymptomatic infection without any associated mortality. ${ }^{43}$ Also, whereas infection of neonates was associated with TUNEL-positive neurons and histological evidence of neuronal death, these findings were absent in 4-week-old brains. A more recent study investigating age-related differences following Coxsackie B5 virus infection in primary cortical neurons from mice also showed substantially more cytotoxicity in immature neurons after infection than cortical neurons, which were matured for 11 days in culture and treated with the same insult. ${ }^{44}$ At this time, little is known about the exact cell death pathways, which may be activated following neuronal exposure to Sindbis and Coxsackie viruses. ${ }^{43,44}$ However, an important theme that can be established from these and other studies described in this section, is that despite the diversity of CNS and PNS subtypes and the various cytotoxic insults presented to neurons both in vitro and in vivo, mature neurons are consistently more resistant to diverse cellular insults.

A noteworthy exception to the phenomenon that mature neurons are more resistant to death than young neurons is the case of excitotoxicity, which occurs when neurons are overstimulated with excitatory compounds, such as glutamate or NMDA. It has been reported that excessive NMDA receptor stimulation causes extensive death in older neurons, while actually favoring increased survival in younger neurons. This topic has been extensively reviewed elsewhere, and the reasons for this phenomenon have not been definitively determined. ${ }^{45}$ Recent findings indicate that changes in NMDA receptor subunit expression with development, increasing intracellular calcium levels with maturation, and changing preference for the ERK versus P38-MAPK pathways as a result of NMDA receptor stimulation as development proceeds may all have a role. ${ }^{46}$

\section{Mechanisms of Enhanced Survival in Mature Neurons}

As neurons are post-mitotic cells with limited regenerative potential, an individual neuron must have the capacity to survive for the organism's entire life, a period that in humans can last a century. Considering that neurons are likely to be exposed to a variety of stresses during this period, one might expect mature neurons to have evolved multiple mechanisms to ensure their long-term survival. Given the longevity of these cells, the presence of redundant brakes would ensure that apoptosis is strictly blocked, even in the event that one safety mechanism fails.

Most of our mechanistic knowledge of this phenomenon comes from experiments performed in sympathetic neurons, where the apoptosis pathway is well characterized. As illustrated in Figure 1, it is now understood that in response to apoptotic stimuli such as NGF deprivation, neonatal

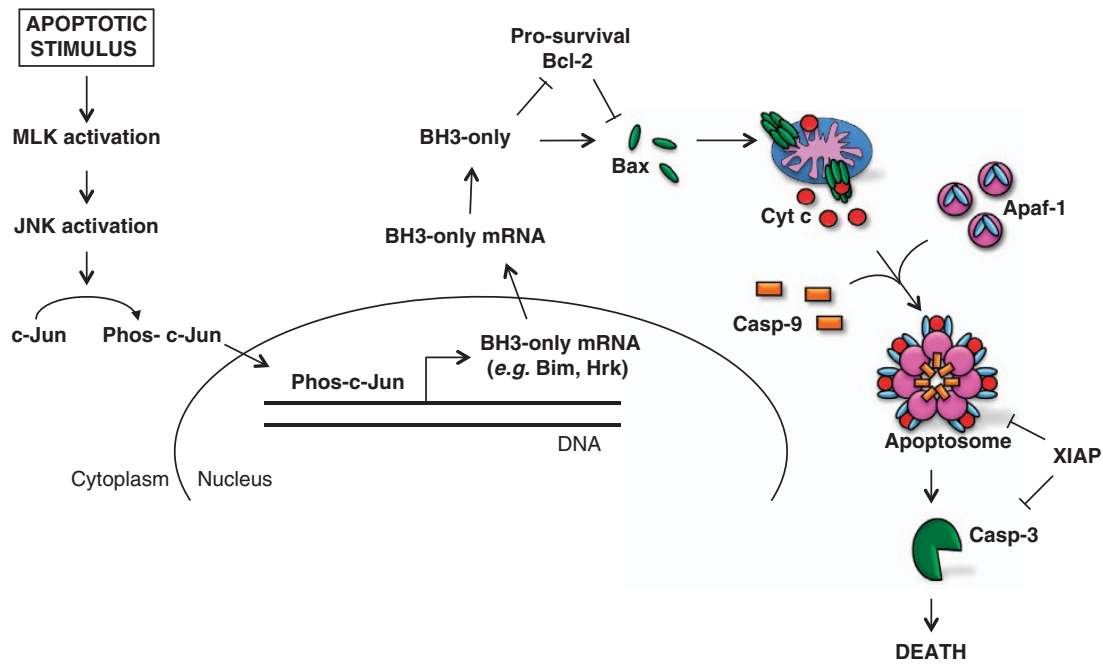

Figure 1 Intrinsic mitochondrial pathway of apoptosis in immature developing neurons. Apoptotic stimuli (e.g., NGF deprivation) initiate a kinase cascade involving MLKs and JNK, which converge on phosphorylation of the transcription factor c-jun. Phosphorylated c-jun (Phos-c-Jun) translocates to the nucleus where it drives transcription of pro-apoptotic BH3-only genes such as Bim and Hrk. The BH3-only family of proteins either directly or indirectly via inhibition of pro-survival Bcl-2 proteins, activate pro-apoptotic Bax via oligomerization and insertion to the outer mitochondrial membrane. Upon mitochondrial permeabilization, cytochrome $c$ (cyt c) is released from the mitochondrial intermembrane space. The presence of cytosolic cytochrome $c$ initiates the formation of the apoptosome complex, which activates caspase- 9 (casp-9) and caspase-3 (casp-3), and ultimately causes cell death 
sympathetic neurons activate the intrinsic mitochondrial pathway of apoptosis. ${ }^{6,19,47-53}$ Briefly, phosphorylation of the transcription factor $\mathrm{c}$-jun by a kinase cascade involving mixed lineage kinase (MLK) and c-Jun N-terminal kinase (JNK) proteins induces the transcription of pro-apoptotic members of the Bcl-2 homology 3 (BH3)-only family of proteins, which mediate the activation of Bax. Active Bax oligomerizes and translocates to the outer mitochondrial membrane, causing its permeabilization and resulting in the release of cytochrome $c$ from the mitochondrial intermembrane space. Cytochrome $c$ then forms the apoptosome complex with apoptotic protease activating factor-1 (Apaf-1) and caspase-9, leading to the cleavage and activation of caspase-3, which in turn cleaves a diverse group of cellular targets to induce rapid cell death.

Pre-mitochondrial apoptotic brakes. The survival of young sympathetic neurons is mediated by the binding of NGF to its tyrosine kinase receptor, TrkA. When NGF is available, TrkA becomes phosphorylated, initiates signaling through the PI-3-kinase/Akt and MEK/MAPK pathways, and is transported retrogradely in a complex with NGF from nerve terminals to the cell body where it promotes neuronal survival. ${ }^{54,55}$ The dephosphorylation of TrkA following NGF withdrawal is one of the first events that initiates apoptosis. Interestingly, studies using compartmentalized culture chambers that allow the separation of neuronal cell bodies from distal axons have shown phospho-TrkA to be significantly more stable in mature neurons following NGF deprivation than in immature counterparts. Specifically, in compartmentalized cultures of immature and mature neurons maintained by exposing NGF only to distal axons, phospho-TrkA can be found in both distal axons and in cell bodies. In response to the removal of NGF, phospho-TrkA in immature neurons becomes undetectable in both the distal axons and cell bodies after just $6 \mathrm{~h}$. In a striking contrast, cell bodies of mature neurons maintain nearly $50 \%$ of phospho-TrkA even after 2 days of NGF deprivation, illustrating a significant delay in the dephosphorylation of TrkA with neuronal age. ${ }^{56}$ As signaling downstream of phospho-TrkA is key to promoting survival in neurons, the sustained phosphorylation of TrkA in mature neurons, even in the absence of NGF, could contribute to their ability to withstand NGF deprivation.

Although the altered kinetics of TrkA dephosphorylation in mature neurons may delay the activation of cell death signals, studies have shown that several of the events immediately downstream of TrkA dephosphorylation still occur in mature neurons. For example, characteristic metabolic changes, JNK activation, and C-Jun phosphorylation all occur in mature neurons after NGF deprivation. ${ }^{18,21}$ Thus, additional and more effective brakes to inhibit apoptosis would be needed downstream of the TrkA receptor to adequately ensure mature neuron survival.

Indeed, studies from the laboratory of Eugene Johnson identified an additional pre-mitochondrial brake to apoptosis downstream of TrkA phosphorylation in mature neurons. NGF deprivation normally activates Bax, which translocates to mitochondria and induces the release of cytochrome $c$ to the cytoplasm in immature neurons. However, immunofluorescence and subcellular fractionation experiments have shown that Bax remains cytoplasmic in mature sympathetic neurons after NGF deprivation. ${ }^{57}$ As a result, cytochrome $c$ remains mitochondrial and is not available in the cytoplasm for subsequent apoptosome formation and caspase activation. In DRG neurons and the rat forebrain, total Bax levels were also found to be decreased with maturation. ${ }^{21,58}$ Consistent with the finding that a block in the apoptotic pathway exists between JNK signaling and Bax activation in mature neurons are data showing that exogenous activation of JNK, via overexpression of MLK3, induces apoptosis less effectively in mature as compared with neonatal DRG neurons. ${ }^{59}$ The inability of mature neurons to activate Bax is extremely effective as a survival mechanism, as Bax deletion has been shown to confer complete resistance of neurons to many intrinsic apoptosis stimuli. ${ }^{52}$

In our own laboratory, we have discovered that a microRNA (miRNA) may also contribute to the inability of Bax to become activated in mature neurons. A microarray analysis to detect differences in miRNA expression between immature and mature neurons showed that miRNA-29 (miR-29) was strikingly upregulated in mature sympathetic neurons both in vivo and in vitro. Interestingly, we found miR-29 to target multiple members of the BH3-only family of genes, which are key proteins necessary for Bax activation during apoptosis. $^{60-62}$ Ectopic expression of miR-29 alone in young sympathetic neurons confers marked resistance to multiple apoptotic stimuli including NGF deprivation, DNA damage, and endoplasmic reticulum stress. Consistent with this model, mature neurons which have high expression of miR-29, do not show induction of $\mathrm{BH}$-family proteins in response to apoptotic stimuli. ${ }^{60}$ Together, these data identified miR-29 as an additional factor that enhances the survival of mature neurons. As miR-29 blocks expression of the multiple, redundant $\mathrm{BH} 3-$ only proteins, this miRNA is able to inhibit apoptosis more potently than the loss of any single $\mathrm{BH} 3-$ only protein alone. Interestingly, a recent report found miR-29 levels to be decreased in a model of acute ischemic stroke caused by middle-cerebral artery occlusion. Importantly, delivering exogenous miR-29 to the cortex reduced the infarct size and improved behavioral outcomes providing evidence that miR-29 can also promote neuroprotection in vivo. ${ }^{63}$

The increased activity of miR-29 in mature neurons may explain the earlier observation that Bax does not translocate to the mitochondria after NGF deprivation in mature neurons. ${ }^{57}$ However, whether restoration of $\mathrm{BH} 3$-only protein expression in mature neurons enables Bax translocation to mitochondria has not been studied. Thus, it remains unclear whether the inability of Bax to translocate to mitochondria is the result of the activity of miR-29 on $\mathrm{BH} 3$-only protein expression, or the result of an independent redundant mechanism blocking Bax translocation.

Post-mitochondrial apoptotic brakes. Even though mature neurons engage mechanisms to inhibit apoptosis upstream of mitochondrial cytochrome $c$ release, they also appear to have evolved additional brakes downstream of mitochondria to further ensure survival. Evidence for this came from experiments involving direct microinjection of cytochrome $c$ protein into cultured neurons. Although immature sympathetic neurons are resistant to cytochrome $c$, 
they can be sensitized to cytochrome $c$ under various conditions. However, even under these conditions, mature neurons remain resistant to cytosolic cytochrome $c{ }^{57}$ For example, although essentially all immature neurons from mice lacking $X$-linked inhibitor of apoptosis protein (XIAP), an endogenous inhibitor of caspase- 9 and caspase- 3 , die within $24 \mathrm{~h}$ of cytosolic cytochrome $c$, mature neurons from XIAP knockout neurons are almost completely resistant. ${ }^{64}$ The survival of mature XIAP knockout neurons to cytochrome $c$ can be attributed to the near-complete loss of Apaf-1 expression in these cells. Apaf-1, a critical scaffolding protein required for cytochrome $c$-mediated caspase activation, is an essential mediator of cell death by apoptosis. Interestingly, multiple neural tissues including the cortex, cerebellum, retina, and sympathetic ganglia have all been found to express limited quantities of Apaf-1. ${ }^{64-68}$ Within the sympathetic neuron model, the lack of Apaf- 1 expression appears to be a result of the greater association of repressed chromatin at the Apaf-1 promoter, resulting in transcriptional silencing. ${ }^{64}$ Similar to Apaf-1, the expression of caspase-3 has been reported to be markedly decreased in multiple neuronal populations of the mature CNS. ${ }^{65,68-70}$ Interestingly, in the motor neuron model, maturation is accompanied with an increase of XIAP and a decrease in its inhibitor XIAPassociated factor (XAF) levels. This increase in the XIAP to XAF ratio increases the ability of XIAP to inhibit caspase- 9 and caspase- 3 and provides mature motor neurons with increased resistance to axotomy-induced apoptosis. ${ }^{71}$

Taken together, the studies highlighted in this section illustrate that mature neurons engage several brakes both upstream and downstream of mitochondrial release of cytochrome $c$, providing redundant mechanisms for strictly regulating apoptosis (Figure 2 , Table 1 ). The inability of cytochrome $c$ to activate caspases in mature neurons is likely to be particularly important for maintaining long-term survival in the event that any mitochondria become damaged and accidentally release cytochrome $c$.

The factors governing the application of these brakes on the apoptotic pathway are currently unknown. As NGF-TrkA signaling is crucial for the development of the sympathetic neuronal population in which many of these brakes have been characterized, NGF-mediated signaling could be important for maturation. However, the fact that phenomena such as Apaf-1 restriction and miR-29 induction have been observed in neuronal populations that do not depend on NGF, such as the cortex and cerebellum, indicates that either growth factor signaling does not have a role in this process, or that other growth factors such as BDNF or GDNF, which have more significant roles in other neuronal populations, can also promote maturation by signaling through a common pathway.

\section{Neurodegeneration: a Return to Immaturity?}

Despite the mechanisms described here that provide neurons with improved capacity to survive injury and apoptosis, adult neurons can still be vulnerable and undergo cell death in situations of acute brain injury or neurodegenerative disease. Why do the changes associated with maturation not protect neurons from neurodegeneration? Are the mechanisms that normally prevent cell death still engaged
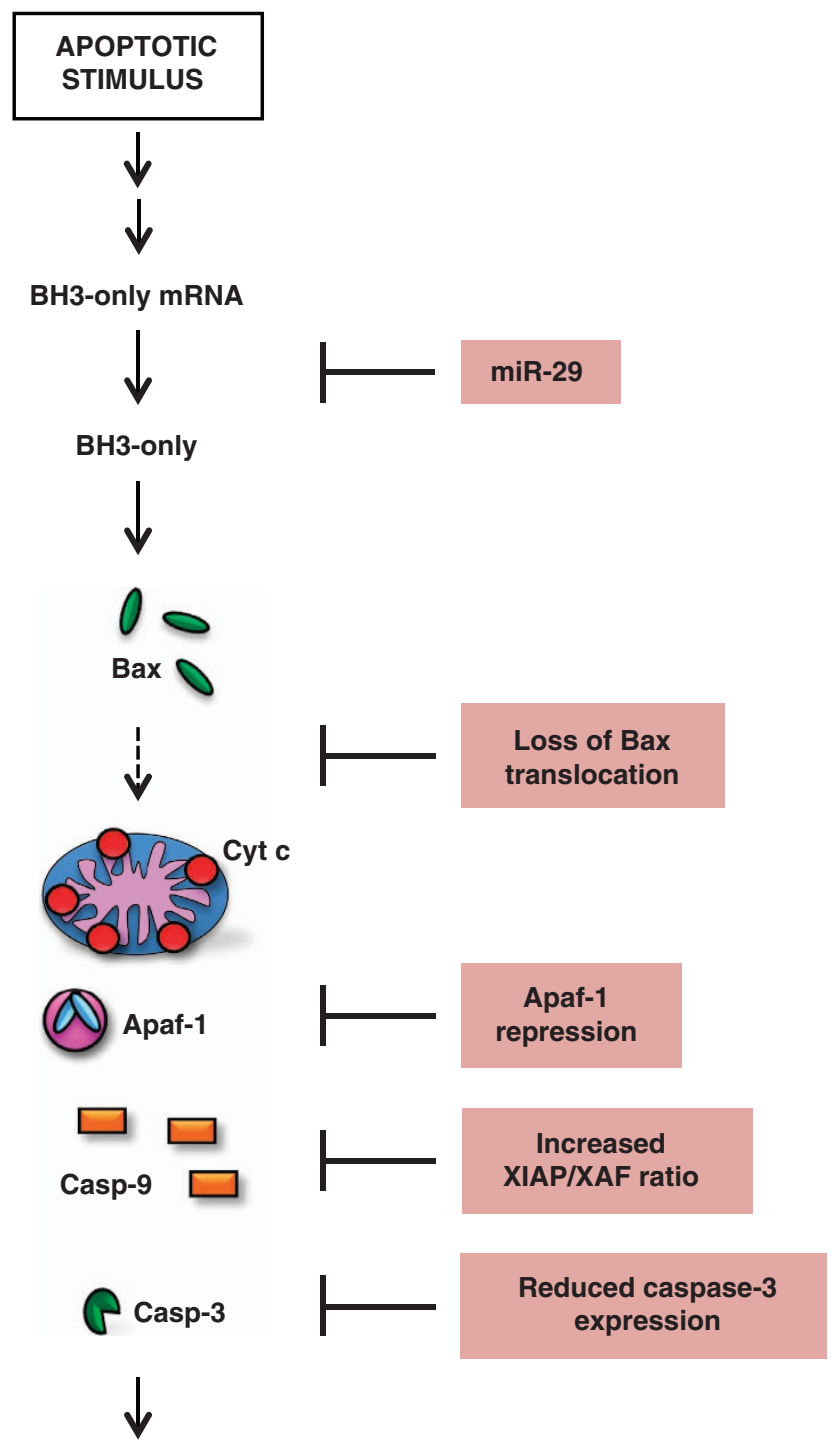

SURVIVAL

Figure 2 Specific mechanisms utilized by mature neurons to inhibit apoptosis. The apoptotic pathway has been found to be inhibited at distinct points in mature neurons. First, multiple neuronal types upregulate the transcription of a microRNA (miR-29), which can target and repress the induction of BH3-only proteins. Second, Bax remains cytosolic in mature neurons and does not translocate to mitochondria, although it is unclear if this is independent of the activity of miR-29 on BH3-only proteins. Third, Apaf-1 expression is repressed via chromatin restriction. Fourth, motor neurons exhibit an increase in the XIAP/XAF ratio, which enables XIAP to more efficiently inhibit both caspase- 9 and caspases-3. Fifth, mature neurons of the CNS have markedly decreased levels of caspases-3. Additional information regarding the specific neuronal types that exhibit these brakes can be found in Table 1

during the degenerative processes in mature neurons? Do the various neurotoxins present in neurodegenerative conditions (e.g., protein aggregates) overwhelm the neurons' survival machinery? Few studies have specifically addressed these questions. Although apoptosis is clearly not the only mechanism mediating neuronal death in the degenerating adult brain, it does appear to contribute to this process. ${ }^{6,72-75}$ 
Here, we highlight emerging evidence that the reversals of events, which promote survival in mature neurons, are seen during neurodegeneration. We speculate that the loss of these survival mechanisms may be a factor that creates a more permissive environment for cell death in these situations of neurodegeneration (Figure 3).

As described previously, one of the mechanisms by which mature neurons inhibit apoptosis is by blocking Bax translocation to mitochondria. Reversal of this protective mechanism in mature neurons would allow adult neurons to regain the ability to translocate Bax to mitochondria, resulting in the release of cytochrome $c$ to the cytosol. Indeed, in mouse models of amyotrophic lateral sclerosis (ALS), where mutant superoxide dismutase-1 (mSOD1) is transgenically overexpressed, both Bax translocation to mitochondria and cytochrome $c$ release are observed as the disease progresses. Compared with non-transgenic controls, Bax levels in spinal cords of symptomatic mSOD1 mice become increasingly more enriched in mitochondrial fractions throughout disease progression. Conversely, cytochrome $c$ levels in cytosolic fractions increase as mice become more symptomatic over time. Cytochrome $c$ translocation was also detected in immunohistochemical studies of human spinal cord from ALS patients. ${ }^{76}$ Similarly, studies of cytochrome $c$ localization have been performed in the context of Huntington's disease (HD). During the most advanced stages of this disease, cytochrome $c$ appears mostly in cytosolic fractions in the neostriatal tissue from human samples. This is in contrast to control tissues where cytochrome $c$ is primarily found in the mitochondrial fraction. ${ }^{77}$ Translocation of cytochrome $c$ from the mitochondria to cytosol was also seen in a mouse model of $\mathrm{HD}$ as the disease progresses. ${ }^{77}$ These data are consistent with Bax-mediated cytochrome $c$ release from mitochondria occurring at a time when neurons are degenerating in ALS and HD. Interestingly, as activation of caspases by cytochrome $c$ is dependent on Apaf- 1 , which is known to be restricted in many mature neuron populations, one would predict that Apaf-1 might be reexpressed during apoptotic neurodegeneration. Although the number of studies that have examined this is limited, Apaf-1 has indeed been found to be reexpressed in mature neurons in response to traumatic brain injury and DNA damage. ${ }^{64,65}$

Table 1 List of known mechanisms by which the apoptotic pathway becomes restricted in mature neurons

\begin{tabular}{|c|c|c|c|}
\hline Target & Neuron/tissue type & Findings & Refs \\
\hline Apaf-1 & $\begin{array}{l}\text { Cortical }^{\mathrm{a}} \\
\text { Cerebellar }^{\mathrm{a}} \\
\text { Sympathetic }^{\mathrm{b}, \mathrm{a}} \\
\text { Photoreceptor }^{\mathrm{b}, \mathrm{a}}\end{array}$ & Decrease in Apaf-1 levels with maturation & $64-68$ \\
\hline BH3-only proteins & Sympathetic $^{b}$ & $\begin{array}{l}\text { Induction of BH3-only family proteins (e.g., Bim, Bmf, Puma, Hrk, N-Bak) is restricted by } \\
\text { miR-29, which is markedly upregulated in mature neurons }\end{array}$ & 60 \\
\hline Bax & $\begin{array}{l}\text { DRG }^{\mathrm{b}, \mathrm{a}} \\
\text { Forebrain }^{\mathrm{a}}\end{array}$ & Decrease in Bax levels with maturation & 21,58 \\
\hline Caspase-3 & $\begin{array}{l}\text { Cortical }^{\mathrm{a}} \\
\text { Photoreceptor }^{\mathrm{b}, \mathrm{a}} \\
\text { Anteroventricular } \\
\text { Cochlear Nucleus }^{\mathrm{a}}\end{array}$ & Shut down of Caspase-3 expression with maturation & $40,65-70$ \\
\hline TrkA & Sympathetic ${ }^{\mathrm{b}}$ & $\begin{array}{l}\text { TrkA phosphorylation persists for a longer period in mature neurons following } \\
\text { NGF deprivation }\end{array}$ & 56 \\
\hline XIAP & Motor $^{\mathrm{a}}$ & $\begin{array}{l}\text { Increase in XIAP/XAF1 ratio enables XIAP to more potently inhibit caspases } \\
\text { in mature neurons }\end{array}$ & 71 \\
\hline
\end{tabular}

aEvidence from in vivo experiments

${ }^{b}$ Evidence from in vitro experiments

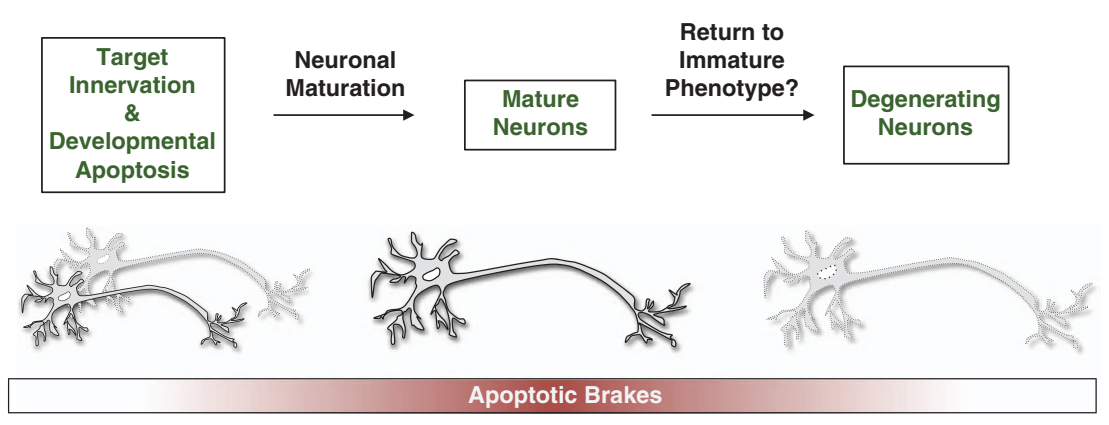

Figure 3 Changes in apoptotic thresholds during neuronal development and neurodegeneration. Schematic shows that developing neurons undergo programmed cell death, a necessary phenomenon for proper nervous system development. Following the developmental period, neurons mature and restrict the apoptotic pathway to permit long-term survival. Under circumstances of neurodegeneration or neuronal injury, the loss of mechanisms to restrict apoptosis causes mature neurons to resemble their immature phenotype 
Another example of an apoptotic brake that may be reversed in degenerating mature neurons comes from studies that have examined miR-29 levels. As discussed earlier, miR-29 exhibits neuroprotective effects in sympathetic neurons by inhibiting the expression of multiple pro-apoptotic $\mathrm{BH} 3-$ only proteins. Although the levels of miR-29 are known to be very high in the various neuronal subtypes of the mature nervous system, ${ }^{60,78}$ miR-29 levels have been reported to be reduced in the striatum of $\mathrm{HD}$ mouse models. ${ }^{79}$ Likewise, the expression of miR-29 was specifically found to be reduced in human brains of Alzheimer's disease patients. ${ }^{78,80}$ Intriguingly, this study also identified $\beta$-site APP-cleaving enzyme 1 (BACE1), a critical mediator of $\beta$-amyloid peptide release from APP, as a target of miR-29. Thus, in addition to the effect on $\mathrm{BH} 3-$ only protein expression, loss of miR-29 expression in sporadic AD could lead to an increase in BACE1 expression and ultimately an increase in $\beta$-amyloid plaques, which are the characteristic protein aggregates of AD. Recently, miR-29 levels have also been reported to be reduced during acute ischemic stroke. ${ }^{63}$ Together, these data support the idea that loss of miR-29 expression, which occurs during neurodegeneration, may increase the vulnerability of mature neurons to progressive degeneration. It should be noted that these observations of reversed apoptotic brakes in neurodegenerative diseases are purely correlative at this point, and more experiments will need to be done to determine the causative role, if any, that these events play in the progression of neurodegenerative disorders.

Although we have focused here on the mitochondrial pathway of apoptosis, studies have found evidence for activation of the death receptor (e.g., Fas) pathway in several neurodegenerative diseases. ${ }^{81,82}$ Whether the death receptor pathway undergoes restriction with neuronal maturation is not known. However, activation of this pathway in mature neurons during neurodegeneration could also circumvent the brakes on the mitochondrial pathway by directly activating caspase-3.

\section{Future Perspective}

Mature neurons have clearly evolved multiple redundant mechanisms that promote long-term neuronal survival. The ability of these cells to withstand a variety of insults is absolutely necessary, especially to limit the risks of neurodegeneration. Moreover, as mature neurons are postmitotic and are not in danger of becoming cancerous, the risk of inhibiting apoptosis in these cells may be minimal to the organism.

Many questions still remain regarding the maturation and enhanced survival of adult neurons. Recent advances in next generation sequencing and proteomics will enable exploration of how the apoptotic pathway changes during neuronal maturation. These changes could include genomic, epigenetic or post-translational modifications. This would expedite the discovery of novel apoptotic brakes or uncover new mechanisms by which the known brakes are regulated during neuronal development and disease. The answers to these and other questions will not only help us to better understand the development and maturation of the nervous system, but also may contribute to our understanding of how neuronal survival unravels during neurodegeneration.

\section{Conflict of Interest}

The authors declare no conflict of interest.

Acknowledgements. We thank members of the Deshmukh lab and Gene Johnson for critical comments. This work was supported by NIH grants NS042197 and GM078366 (to MD). AJK was supported by grants T32GM008719 and F30NS068006

1. Oppenheim RW. Cell death during development of the nervous system. Annu Rev Neurosci 1991; 14: 453-501.

2. Kim WR, Sun W. Programmed cell death during postnatal development of the rodent nervous system. Dev Growth Differ 2011; 53: 225-235.

3. Davies AM. Regulation of neuronal survival and death by extracellular signals during development. EMBO J 2003; 22: 2537-2545

4. Buss RR, Sun W, Oppenheim RW. Adaptive roles of programmed cell death during nervous system development. Annu Rev Neurosci 2006; 29: 1-35.

5. Benn SC, Woolf CJ. Adult neuron survival strategies-slamming on the brakes. Nat Rev Neurosci 2004; 5: 686-700.

6. Yuan J, Yankner BA. Apoptosis in the nervous system. Nature 2000; 407: 802-809.

7. Galluzzi L, Blomgren K, Kroemer G. Mitochondrial membrane permeabilization in neuronal injury. Nat Rev Neurosci 2009; 10: 481-494.

8. Ernst M. Ueber Untergang von Zellen wahrend der normalen Entwicklung bei Wirbeltieren. Zeitschr Anat u Entw gesch 1926; 79: 228-262.

9. Glucksmann A. Ueber die Bedeutung von Zellvorgangen fur die Formbildung epithelialer Organe. Zeitschr Anat u Entw gesch 1930; 93: 35-92.

10. Hamburger V, Levi-Montalcini R. Proliferation, differentiation and degeneration in the spinal ganglia of the chick embryo under normal and experimental conditions. J Exp Zool 1949; 111: 457-501.

11. Levi-Montalcini $R$, Booker $B$. Destruction of the sympathetic ganglia in mammals by an antiserum to a nerve-growth protein. Proc Natl Acad Sci USA 1960; 46: 384-391.

12. Angeletti PU, Levi-Montalcini R, Caramia F. Analysis of the effects of the antiserum to the nerve growth factor in adult mice. Brain Res 1971; 27: 343-355.

13. Goedert M, Otten U, Thoenen $\mathrm{H}$. Biochemical effects of antibodies against nerve growth factor on developing and differentiated sympathetic ganglia. Brain Res 1978; 148: 264-268.

14. Otten U, Goedert M, Schwab M, Thibault J. Immunization of adult rats against 2.5 S NGF: effects on the peripheral sympathetic nervous system. Brain Res 1979; 176: 79-90.

15. Gorin PD, Johnson EM Jr. Effects of long-term nerve growth factor deprivation on the nervous system of the adult rat: an experimental autoimmune approach. Brain Res 1980; 198: $27-42$.

16. Lazarus KJ, Bradshaw RA, West NR, Bunge P. Adaptive survival or rat sympathetic neurons cultured without supporting cells or exogenous nerve growth factor. Brain Res 1976; 113: 159-164.

17. Chun LL, Patterson PH. Role of nerve growth factor in the development of rat sympathetic neurons in vitro. II. Developmental studies. J Cell Biol 1977; 75: 705-711.

18. Easton RM, Deckwerth TL, Sh PA, Johnson EM Jr. Analysis of the mechanism of loss of trophic factor dependence associated with neuronal maturation: A phenotype indistinguishable from BAX deletion. J Neurosci 1997; 17: 9656-9666.

19. Deshmukh M, Johnson EM Jr. Programmed cell death in neurons: focus on the pathway of nerve growth factor deprivation-induced death of sympathetic neurons. Mol Pharm 1997; 51: 897-906.

20. Freeman RS, Burch RL, Crowder RJ, Lomb DJ, Schoell MC, Straub JA et al. NGF deprivation-induced gene expression: after ten years, where do we stand? Prog Brain Res 2004; 146: 111-126.

21. Vogelbaum MA, Tong JX, Rich KM. Developmental regulation of apoptosis in dorsal root ganglion neurons. J Neurosci 1998; 18: 8928-8935.

22. Kimpinski K, Campenot RB, Mearow K. Effects of the neurotrophins nerve growth factor neurotrophin-3, and brain-derived neurotrophic factor (BDNF) on neurite growth from adult sensory neurons in compartmented cultures. J Neurobiol 1997; 33: 395-410.

23. Lindsay RM. Nerve growth factors (NGF, BDNF) enhance axonal regeneration but are not required for survival of adult sensory neurons. J Neurosci 1988; 8: 2394-2405.

24. Tong JX, Eichler ME, Rich KM. Intracellular calcium levels influence apoptosis in mature sensory neurons after trophic factor deprivation. Exp Neurol 1996; 138: 45-52.

25. Harris JA, Rubel EW. Afferent regulation of neuron number in the cochlear nucleus: cellular and molecular analyses of a critical period. Hear Res 2006; 216-217: 127-137.

26. Mostafapour SP, Del Puerto NM, Rubel EW. bcl-2 Overexpression eliminates deprivationinduced cell death of brainstem auditory neurons. J Neurosci 2002; 22: 4670-4674.

27. Tierney TS, Russell FA, Moore DR. Susceptibility of developing cochlear nucleus neurons to deafferentation-induced death abruptly ends just before the onset of hearing. J Comp Neurol 1997; 378: 295-306.

28. Mostafapour SP, Cochran SL, Del Puerto NM, Rubel EW. Patterns of cell death in mouse anteroventral cochlear nucleus neurons after unilateral cochlea removal. J Comp Neurol 2000; 426: 561-571.

29. Snider WD, Elliott JL, Yan Q. Axotomy-induced neuronal death during development. J Neurobiol 1992; 23: 1231-1246. 
30. Snider WD, Thanedar S. Target dependence of hypoglossal motor neurons during development in maturity. J Comp Neurol 1989; 279: 489-498.

31. Yan Q, Snider WD, Pinzone JJ, Johnson EM Jr. Retrograde transport of nerve growth factor (NGF) in motoneurons of developing rats: assessment of potential neurotrophic effects. Neuron 1988; 1: 335-343.

32. Sendtner M, Kreutzberg GW, Thoenen $H$. Ciliary neurotrophic factor prevents the degeneration of motor neurons after axotomy. Nature 1990; 345: 440-441.

33. Yu WH. Sex difference in neuronal loss induced by axotomy in the rat brain stem motor nuclei. Exp Neurol 1988; 102: 230-235.

34. Kuzis K, Coffin JD, Eckenstein FP. Time course and age dependence of motor neuron death following facial nerve crush injury: role of fibroblast growth factor. Exp Neurol 1999; 157: $77-87$

35. Rich KM, Luszczynski JR, Osborne PA, Johnson EM Jr. Nerve growth factor protects adult sensory neurons from cell death and atrophy caused by nerve injury. J Neurocytol 1987; 16: 261-268.

36. McKernan DP, Caplis C, Donovan M, O'Brien CJ, Cotter TG. Age-dependent susceptibility of the retinal ganglion cell layer to cell death. Invest Ophthalmol Vis Sci2006; 47: 807-814.

37. Guerin MB, McKernan DP, O'Brien CJ, Cotter TG. Retinal ganglion cells: dying to survive. Int J Dev Biol 2006; 50: 665-674.

38. Bittigau P, Sifringer M, Pohl D, Stadthaus D, Ishimaru M, Shimizu H et al. Apoptotic neurodegeneration following trauma is markedly enhanced in the immature brain. Ann Neurol 1999; 45: 724-735.

39. Cheng Y, Deshmukh M, D'Costa A, DeMaro JA, Gidday JM, Shah A et al. Caspase inhibitor affords neuroprotection with delayed administration in a rat model of neonatal hypoxic-ischemic brain injury. J Clin Invest 1998; 101: 1992-1999.

40. Hu BR, Liu CL, Ouyang Y, Blomgren K, Siesjo BK. Involvement of Caspase-3 in Cell Death After Hypoxia-Ischemia Declines During Brain Maturation. J Cereb Blood Flow Metab 2000; 20: 1294-1300.

41. Liu CL, Siesjo BK, Hu BR. Pathogenesis of hippocampal neuronal death after hypoxiaischemia changes during brain development. Neuroscience 2004; 127: 113-123.

42. Blomgren K, Zhu C, Hallin U, Hagberg H. Mitochondria and ischemic reperfusion damage in the adult and in the developing brain. Biochem Biophys Res Commun 2003; 304: $551-559$

43. Labrada L, Liang XH, Zheng W, Johnston C, Levine B. Age-dependent resistance to lethal alphavirus encephalitis in mice: analysis of gene expression in the central nervous system and identification of a novel interferon-inducible protective gene, mouse ISG12. J Virol 2002; 76: 11688-11703

44. Ahn J, Jee Y, Seo I, Yoon SY, Kim D, Kim YK et al. Primary neurons become less susceptible to coxsackievirus B5 following maturation: the correlation with the decreased level of CAR expression on cell surface. J Med Virol 2008; 80: 434-440.

45. Hetman M, Kharebava G. Survival signaling pathways activated by NMDA receptors. Curr Top Med Chem 2006; 6: 787-799.

46. Xiao L, Hu C, Feng C, Chen Y. Switching of N-methyl-D-aspartate (NMDA) receptorfavorite intracellular signal pathways from ERK $1 / 2$ protein to $\mathrm{p} 38$ mitogen-activated protein kinase leads to developmental changes in NMDA neurotoxicity. J Biol Chem 2011; 286: 20175-20193.

47. Mota M, Reeder M, Chernoff J, Bazenet CE. Evidence for a role of mixed lineage kinases in neuronal apoptosis. J Neurosci 2001; 21: 4949-4957.

48. Xu Z, Maroney AC, Dobrzanski P, Kukekov NV, Greene LA. The MLK family mediates C-Jun N-terminal kinase activation in neuronal apoptosis. Mol Cell Biol 2001; 21: 4713-4724.

49. Imaizumi K, Tsuda M, Imai Y, Wanaka A, Takagi T, Tohyama M. Molecular cloning of a novel polypeptide, DP5, induced during programmed neuronal death. J Biol Chem 1997; 272: $18842-18848$

50. Harris CA, Johnson EM Jr.. BH3-only Bcl-2 family members are coordinately regulated by the JNK pathway and require Bax to induce apoptosis in neurons. J Biol Chem 2001; 276: 37754-37760

51. Whitfield J, Neame SJ, Paquet L, Bernard O, Ham J. Dominant-negative c-Jun promotes neuronal survival by reducing BIM expression and inhibiting mitochondrial cytochrome $c$ release. Neuron 2001; 29: 629-643.

52. Deckwerth TL, Elliott JL, Knudson CM, Johnson EM Jr, Snider WD, Korsmeyer SJ. Bax is required for neuronal death after trophic factor deprivation and during development. Neuron 1996; 17: 401-411.

53. Wright KM, Vaughn AE, Deshmukh M. Apoptosome dependent caspase-3 activation pathway is non-redundant and necessary for apoptosis in sympathetic neurons. Cell Death Differ 2007; 14: 625-633.

54. Snider WD. Functions of the neurotrophins during nervous system development: what the knockouts are teaching us. Cell 1994; 77: 627-638.

55. Kaplan DR, Miller FD. Neurotrophin signal transduction in the nervous system. Curr Opin Neurobiol 2000; 10: 381-391.

56. Tsui-Pierchala BA, Ginty DD. Characterization of an NGF-P-TrkA retrograde-signaling complex and age-dependent regulation of TrkA phosphorylation in sympathetic neurons. J Neurosci 1999; 19: 8207-8218.

57. Putcha GV, Deshmukh M, Johnson EM Jr. Inhibition of apoptotic signaling cascades causes loss of trophic factor dependence during neuronal maturation. J Cell Biol 2000; 149: $1011-1018$
58. Polster BM, Robertson CL, Bucci CJ, Suzuki M, Fiskum G. Postnatal brain development and neural cell differentiation modulate mitochondrial $\mathrm{Bax}$ and $\mathrm{BH} 3$ peptide-induced cytochrome c release. Cell Death Differ 2003; 10: 365-370.

59. Walsh GS, Orike N, Kaplan DR, Miller FD. The invulnerability of adult neurons: a critical role for p73. J Neurosci 2004; 24: 9638-9647.

60. Kole AJ, Swahari V, Hammond SM, Deshmukh M. miR-29b is activated during neuronal maturation and targets BH3-only genes to restrict apoptosis. Genes Dev 2011; 25: $125-130$

61. Giam M, Huang DC, Bouillet P. BH3-only proteins and their roles in programmed cell death Oncogene 2008; 27(Suppl 1): S128-S136.

62. Lomonosova $\mathrm{E}$, Chinnadurai $\mathrm{G}$. BH3-only proteins in apoptosis and beyond: an overview. Oncogene 2008; 27(Suppl 1): S2-S19.

63. Khanna S, Rink C, Ghoorkhanian R, Gnyawali S, Heigel M, Wijesinghe DS et al. Loss of miR-29b following acute ischemic stroke contributes to neural cell death and infarct size. J Cereb Blood Flow Metab 2013; e-pub ahead of print 1 May 2013; doi:10.1038/ jcbfm.2013.68.

64. Wright KM, Smith MI, Farrag L, Deshmukh M. Chromatin modification of Apaf-1 restricts the apoptotic pathway in mature neurons. J Cell Biol 2007; 179: 825-832.

65. Yakovlev AG, Ota K, Wang G, Movsesyan V, Bao W-L, Yoshihara K et al. Differential expression of apoptotic protease-activating factor-1 and caspase-3 genes and susceptibility to apoptosis during brain development and after traumatic brain injury. J Neurosci 2001; 21: 7439-7446.

66. Johnson $C E$, Huang $Y Y$, Parrish $A B$, Smith MI, Vaughn $A E$, Zhang $Q$ et al. Differential Apaf-1 levels allow cytochrome $c$ to induce apoptosis in brain tumors but not in normal neural tissues. Proc Natl Acad Sci USA 2007; 104: 20820-20825.

67. Donovan M, Cotter TG. Caspase-independent photoreceptor apoptosis in vivo and differential expression of apoptotic protease activating factor- 1 and caspase- 3 during retinal development. Cell Death Differ 2002; 9: 1220-1231.

68. Ota K, Yakovlev AG, Itaya A, Kameoka M, Tanaka Y, Yoshihara K. Alteration of apoptotic protease-activating factor-1 (APAF-1)-dependent apoptotic pathway during development of rat brain and liver. J Biochem 2002; 131: 131-135.

69. Blomgren K, Zhu C, Wang X, Karlsson JO, Leverin AL, Bahr BA et al. Synergistic activation of caspase-3 by m-calpain after neonatal hypoxia-ischemia: a mechanism of 'pathological apoptosis'? J Biol Chem 2001; 276: 10191-10198.

70. Harris JA, Hardie NA, Bermingham-McDonogh O, Rubel EW. Gene expression differences over a critical period of afferent-dependent neuron survival in the mouse auditory brainstem. J Comp Neurol 2005; 493: 460-474.

71. Perrelet D, Perrin FE, Liston P, Korneluk RG, MacKenzie A, Ferrer-Alcon M et al. Motoneuron resistance to apoptotic cell death in vivo correlates with the ratio between X-linked inhibitor of apoptosis proteins (XIAPs) and its inhibitor, XIAP-associated factor 1. J Neurosci 2004; 24: 3777-3785.

72. Chan PH. Mitochondria and neuronal death/survival signaling pathways in cerebral ischemia. Neurochem Res 2004; 29: 1943-1949.

73. Martin LJ, Al-Abdulla NA, Brambrink AM, Kirsch JR, Sieber FE, Portera-Cailliau C. Neurodegeneration in excitotoxicity, global cerebral ischemia, and target deprivation: a perspective on the contributions of apoptosis and necrosis. Brain Res Bull 1998; 46: 281-309.

74. Mattson MP. Apoptosis in neurodegenerative disorders. Nat Rev Mol Cell Biol 2000; 120-129.

75. Choi DW. Ischemia-induced neuronal apoptosis. Curr Opin Neurobiol 1996; 6: 667-672.

76. Guegan C, Vila M, Rosoklija G, Hays AP, Przedborski S. Recruitment of the mitochondrialdependent apoptotic pathway in amyotrophic lateral sclerosis. J Neurosci 2001; 21: 6569-6576.

77. Kiechle T, Dedeoglu A, Kubilus J, Kowall NW, Beal MF, Friedlander RM et al. Cytochrome $\mathrm{C}$ and caspase-9 expression in Huntington's disease. Neuromolecular Med 2002; 1: 183-195.

78. Hebert SS, Horre K, Nicolai L, Papadopoulou AS, Mandemakers W, Silahtaroglu AN et al. Loss of microRNA cluster miR-29a/b-1 in sporadic Alzheimer's disease correlates with increased BACE1/beta-secretase expression. Proc Natl Acad Sci USA 2008; 105 6415-6420.

79. Lee ST, Chu K, Im WS, Yoon HJ, Im JY, Park JE et al. Altered microRNA regulation in Huntington's disease models. Exp Neurol 2011; 227: 172-179.

80. Shioya M, Obayashi S, Tabunoki H, Arima K, Saito Y, Ishida T et al. Aberrant microRNA expression in the brains of neurodegenerative diseases: miR-29a decreased in Alzheimer disease brains targets neurone navigator 3. Neuropathol Appl Neurobiol 2010; 36: 320-330.

81. Choi C, Benveniste EN. Fas ligand/Fas system in the brain: regulator of immune and apoptotic responses. Brain Res Rev 2004; 44: 65-81.

82. Haase G, Pettmann B, Raoul C, Henderson CE. Signaling by death receptors in the nervous system. Curr Opin Neurobiol 2008; 18: 284-291.

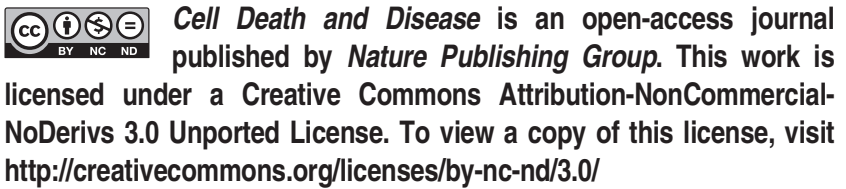

